\title{
Multidisciplinary Design under Uncertainty for a Hypersonic Vehicle
}

\author{
Geng Zhang ${ }^{1}$ and Jim $\mathrm{He}^{2}$ \\ Michigan Engineering Services, LLC, Ann Arbor, MI, 48108 \\ Nickolas Vlahopoulos ${ }^{3}$ \\ University of Michigan, Ann Arbor, MI, 48109
}

\begin{abstract}
An integration environment has been developed for conducting multidiscipline design optimization analysis under uncertainty. It facilitates solution of multiple optimization problems in parallel with multiple sets of objectives and constraints originating from different design disciplines while simultaneously accounting for uncertainty during the optimization process. A seamless general purpose integration capability facilitates exchanging data between the optimization processes and the solvers which are used for evaluating the objective functions and the constraints. Metamodels can be developed and used instead of the actual solvers during the highly iterative optimization process in order to expedite the computations. Uncertainties are introduced in the optimization by considering the constraints which depend on any random variables and any random parameters as probabilistic. Satisfying the probabilistic constraints in the presence of uncertainty introduces sufficient conservatism in the solution and eliminates the need for further application of safety factors. The work presented in this paper considers trajectory, aerothermal, aerodynamic, thermal, and structural computations when performing the design optimization for the Thermal Protection System (TPS) and for the structure of a TSTO upper stage vehicle. Sixteen different sections are considered on the vehicle when designing the TPS. The trajectory bank angle schedule, the angle of attack schedule, the thickness of the sixteen different TPS sections, and twenty seven thicknesses associated with the structure are considered when reducing the overall weight of the vehicle while satisfying the imposed constraints. Uncertainties are considered in three control angles of the trajectory, in the material strength, the thrust load and the 2.5G loads. The results from the multi-discipline optimization without and with uncertainty are discussed, and a comparison between the deterministic and the probabilistic optimum is made.
\end{abstract}

\section{Introduction}

$\mathrm{T}$ HE physical difficulty of designing entry vehicles originates from the large degree of coupling between the various disciplines involved in the design [1-3]. The disciplines which must be accounted for and integrated during the design are: trajectory optimization [4-6], guidance, navigation, and control (GN\&C) technology [7,8], aerodynamics and aerothermodynamics [9-11], thermal-structural analysis [12-14], and thermal protection system (TPS) development [15-19]. Efforts have been made in developing a collaborative or a multidisciplinary optimization process that considers some of the disciplines of interest during an integrated design [20-23]. Further, it is important to capture in the decision making process how uncertainty in the atmospheric conditions, in the entry parameters of the vehicle, in the condition of the vehicle during entry, and in the performance of the TPS will influence the design and provide a risk assessment for a mission. The work presented in this paper considers trajectory, aerothermal, aerodynamic, thermal, and structural computations when performing the design optimization for the Thermal Protection System (TPS) and for the structure of a TSTO upper stage vehicle. It utilizes an integration framework developed and presented previously [24, 25] for conducting multidiscipline design optimization (MDO) under uncertainty. Sixteen different sections are considered on the vehicle when designing the

\footnotetext{
${ }^{1}$ Research and Development Engineer, 2890 Carpenter Road, Suite 1900, Ann Arbor, MI 48108.

${ }^{2}$ Research and Development Engineer, 2890 Carpenter Road, Suite 1900, Ann Arbor, MI 48108.

${ }^{3}$ Professor, NA\&ME Depat., 2600 Draper Road, Ann Arbor, MI 48109, AIAA Member.
} 
TPS. The trajectory bank angle schedule, the angle of attack schedule, the thickness of the sixteen different TPS sections, and twenty seven thicknesses associated with the structure are used as design variables in order to reduce the overall weight of the vehicle while satisfying the imposed constraints. Both a deterministic and a probabilistic analysis is conducted. In the latter, uncertainties are considered in three control angles of the trajectory, in the material strength, the thrust load and the $2.5 \mathrm{G}$ loads.

The trajectory code Traj [26] developed at NASA Ames is employed for the trajectory computations. Aerothermal information is computed at each point of the trajectory from flight characteristics computed by Traj (Mach number, angle of attack, and dynamic pressure) and an aerothermal database provided by NASA Ames and created using the (configuration Based AEROdynamics) CBAERO package $[27,28]$. The CBAERO code has been utilized in the past for designing the trajectory and the thermal protection system for reusable launch vehicles [29]. In this application the trajectory is controlled by an angle of attack schedule containing seven angles, and a bank angle schedule containing seven angles.

Thermal analyses are performed for each TPS material system corresponding to each of the sixteen representative locations. The thermal loads are determined from the trajectory results and the aerothermal database. The FIAT code developed at NASA Ames [30] is employed in the thermal computations. The thermal optimization is minimizing the thickness of the TPS at sixteen different locations. The thermal environments are evaluated from the Mach number, angle of attack, and dynamic pressure time histories. Soak out conditions are considered in this analysis. Constraints are imposed at temperatures on the bondline surface and locations within the graphite and the core material.

The structural optimization minimizes the weight of the structure while considering four loading conditions: loads corresponding to $\max (\mathrm{Q}), \max (\mathrm{Q} a \mathrm{lph})$, the $2.5 \mathrm{G}$ load, and the thrust load. The first two depend on the trajectory while the last two are independent of the trajectory and are constant for all different trajectory configurations. Twenty seven design variables linked with the thickness of the graphite layers and the thickness of the aluminum core are considered in the structural optimization. The MSC/Nastran SOL200 code is used for conducting the structural optimization. Two important issues associated with the structural optimization shaped the manner that the overall multi-discipline optimization analysis is conducted. The thrust load and the $2.5 \mathrm{G}$ loads dominate the solution of the structural optimization. Thus, the trajectory results and the related aerodynamic loads do not influence the outcome of the structural optimization in this particular application. Additionally, the starting point for the structural design variables leads to different results in the NASTRAN SOL200. In order to address these two issues the following actions were taken: The structural optimization was decoupled from the rest of the disciplines (trajectory and thermal); and a customized algorithm is developed and used for the structural optimization in order to address the dependency of the optimal solution to the starting point for the optimization.

The formulation of the multi-discipline optimization analysis, and the results from the multi-discipline optimization without and with uncertainty are discussed, and a comparison between the deterministic and the probabilistic optimum is made.

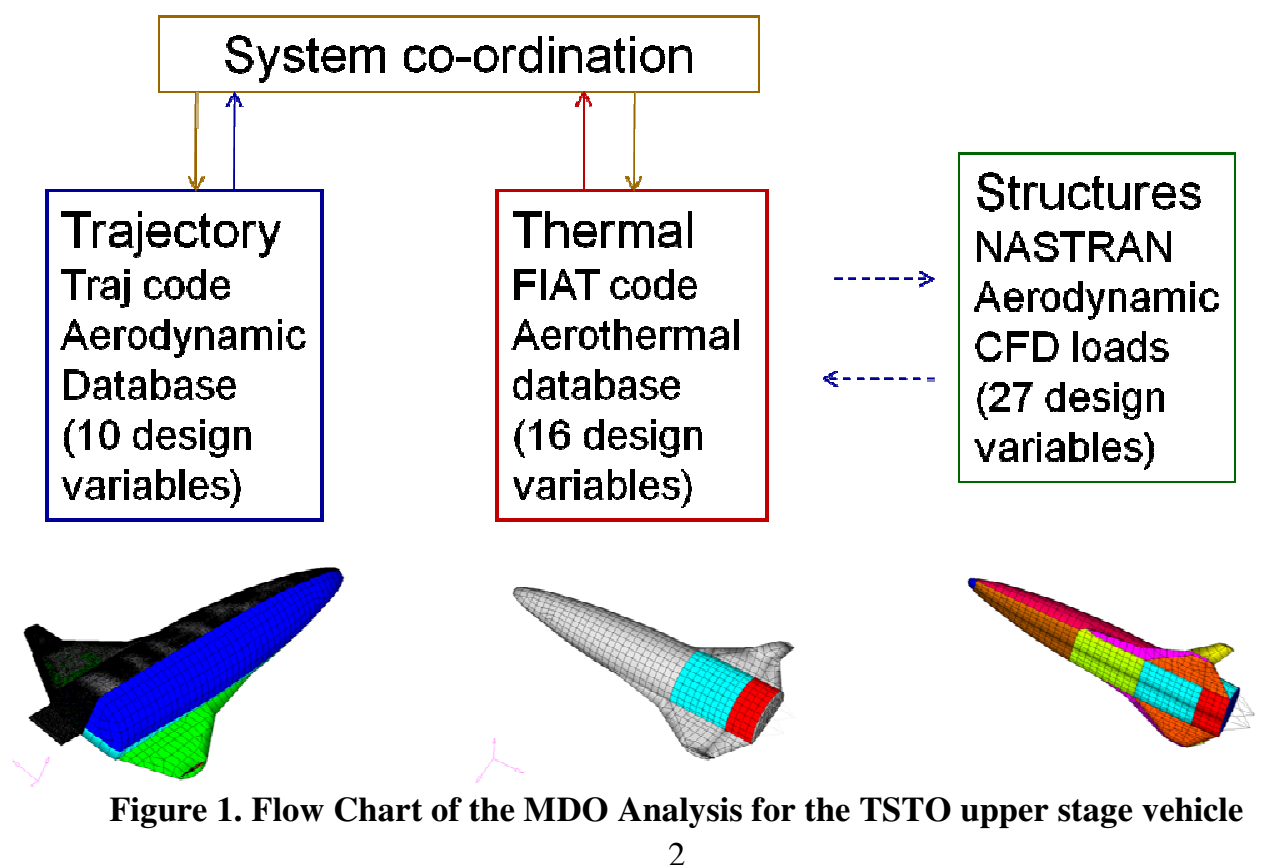

American Institute of Aeronautics and Astronautics 


\section{Optimization Statements for the Multidiscipline Analysis}

The flow chart of the multi-discipline optimization analysis is presented in Figure 1. Once it was realized that the trajectory dependent aerodynamic loads (pressure loads for $\max (\mathrm{Q})$ and $\max (\mathrm{Q} a \mathrm{lph} a)$ ) have no impact on the results of the structural optimization the structural optimization was removed from the tight integration of the multidiscipline analysis. This is necessary because the top level optimizer gets no response to any attempts to influence the outcome of the structural optimization and thus it cannot operate properly. The results from the optimal thickness of the structure are taken into account in the FIAT thermal analysis. Since the $\max (\mathrm{Q})$ and $\max (\mathrm{Q} a \mathrm{lph}$ ) loads from the trajectory have no influence on the optimal configuration of the structure, no attempts are made to establish any iterative process between the structural optimization and the remaining two disciplines. The optimal structural configuration is determined first and then the rest of the analysis is conducted. This is necessary because the structural skin thickness influences the results of the thermal analysis.

\section{A. Trajectory Optimization}

Through a variable screening process ten design variables are defined from the trajectory analysis out of the seven angle of attacks and eleven bank angles. The time schedule for the angles of attack and bank angles is summarized in Figure 2. The angles that are selected as design variables for the trajectory optimization are also identified in Figure 2.

\section{Bank Angles}

\section{Angles of Attack}

\begin{tabular}{|l|r|}
\hline$\ddot{*}$ & Time (sec) \\
\hline 1 & 0.0 \\
\hline 2 & 500.0 \\
\hline 3 & 1000.0 \\
\hline 4 & 1500.0 \\
\hline 5 & 2000.0 \\
\hline 6 & 2500.0 \\
\hline 7 & 3000.0 \\
\hline
\end{tabular}

\begin{tabular}{|l|r|}
\hline$\ddot{\#}$ & Time (sec) \\
\hline 1 & 0.0 \\
\hline 2 & 250.0 \\
\hline 3 & 500.0 \\
\hline 4 & 750.0 \\
\hline 5 & 1000.0 \\
\hline 6 & 1250.0 \\
\hline 7 & 1500.0 \\
\hline 8 & 1750.0 \\
\hline 9 & 2000.0 \\
\hline 10 & 2500.0 \\
\hline 11 & 3000.0 \\
\hline
\end{tabular}

Figure 2. Schedule of angles of attack schedule and bank angles schedule; identification of design variables for trajectory analysis

The Traj code is utilized as the solver during the trajectory optimization analysis. An aerodynamic and an aerothermal database are linked with the Traj code in order to generate the necessary information for Traj. The high speed aerodynamic and aerothermodynamic databases for the TSTO Upper Stage vehicle are generated using the CBAERO code. CBAERO is an engineering-based code for estimating the forces and aero heating on the surface of a reentry vehicle. Surface pressures are computed using independent panel methods (e.g. Newtonian Flow). Surface shear forces and convective heating are computed using Reference Enthalpy methods for acreage areas and Fay-Riddell methods for stagnation regions. Aerodynamic coefficients used in the trajectory analysis are computed by integrating pressure and shear forces over the surface of the vehicle to obtain trimmed aerodynamic lift and drag coefficients as function of flight Mach number, angle-of-attack and free-stream dynamic pressure.

The objective function of the trajectory optimization is to minimize the heat load. At the same time the following constraints are imposed:

$\operatorname{Max} \mathrm{G}<3$

$\operatorname{Max} \mathrm{Q}(\mathrm{psf})<500.0$

Max QA (psf-degrees) $<8000.0$

Cross Range $(\mathrm{nm})>1200.0$

Limits on maximum temperature at 16 body points (Figure 3 and Table 1) 
Maximum temperature limits are imposed on sixteen body points of the TSTO vehicle, the location of each point, the type of thermal protection material used, and the corresponding maximum temperature limit are summarized in Table 1. Additionally, the sixteen points are presented in Figure 3 (note that some points in Figure 3 are related with the same physical location of the vehicle). The actual Traj solver is utilized in the trajectory optimization simulations.

\begin{tabular}{|c|c|c|c|c|}
\hline BP\# & $\underline{\text { Trï\# }}$ & Location & Material & $\underline{\mathrm{T}}_{\text {LIMIT }}\left({ }^{\circ} \mathrm{K}\right)$ \\
\hline 1 & None & Nosecap Stagnation & $\mathrm{ACC}$ & 2000 \\
\hline 2 & 33506 & $10 \%$ Body Wind & AETB/TUFI & 1650 \\
\hline 3 & 43219 & $25 \%$ Body Wind & CRI & 1400 \\
\hline 4 & 35179 & $50 \%$ Body Wind & CRI & 1400 \\
\hline 5 & 35806 & $75 \%$ Body Wind & CRI & 1400 \\
\hline 6 & 24080 & $10 \%$ Body Lee & CRI & 1400 \\
\hline 7 & 24834 & $25 \%$ Body Lee & FRSI & 750 \\
\hline 8 & 25775 & $50 \%$ Body Lee & FRSI & 750 \\
\hline 9 & 8624 & Wing L/E & $\mathrm{ACC}$ & 2000 \\
\hline 10 & 9742 & Wing L/E & $\mathrm{ACC}$ & 2000 \\
\hline 11 & 16785 & Wing $1 / 4 \mathrm{C}$ Wind & AETB/TUFI & 1650 \\
\hline 12 & 10645 & Strake & AETB/TUFI & 1650 \\
\hline 13 & 2054 & Winglet L/E & AETB/TUFI & 1650 \\
\hline 14 & 37383 & Wing $50 \% \mathrm{C}$ Lee & FRSI & 750 \\
\hline 15 & 16785 & Wing $1 / 4 \mathrm{C}$ Wind & AETB/TUFI & 1650 \\
\hline 16 & 16840 & Wing $50 \% \mathrm{C}$ Wind & CRI & 1400 \\
\hline
\end{tabular}
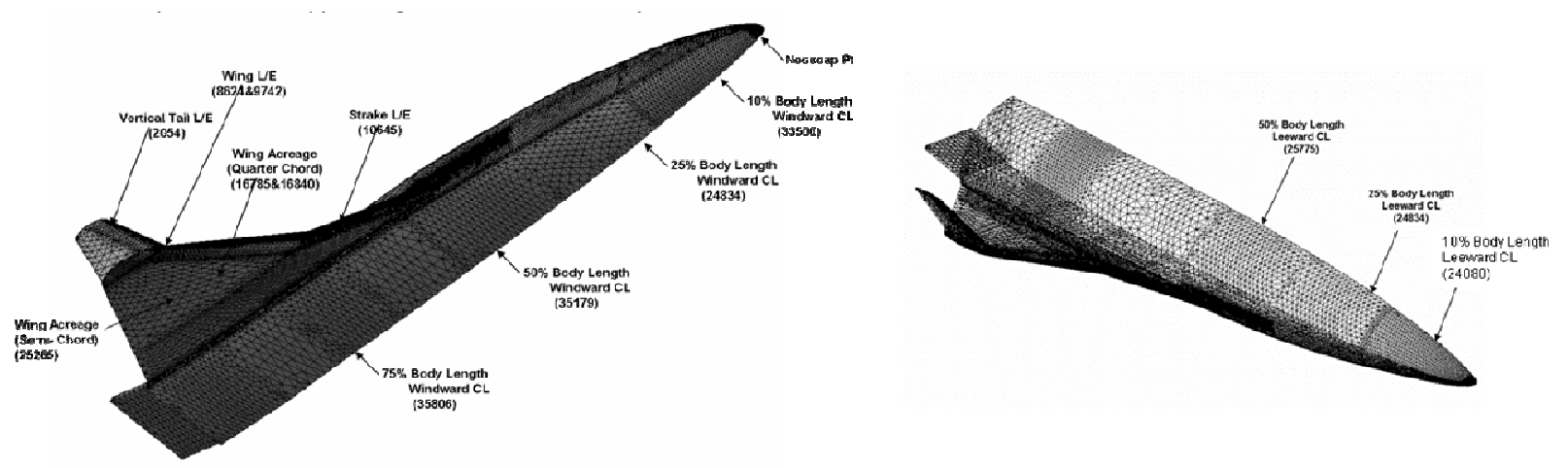

Figure 3. Summary of sixteen body points defining the thermal constraints in the trajectory optimization, and the locations where thermal optimization analysis is performed

\section{B. Thermal Protection System Optimization}

In the thermal discipline the objective is to minimize the total mass of the TPS. Sixteen design variables (the thickness of the thermal protection associated with each one of the sixteen body points) are considered in this analysis. Table 2 summarizes the sixteen body points, the area where they are applied, the material used, and the total area associated with each location. 


\begin{tabular}{|c|c|c|c|c|c|}
\hline BP\# & Tri \# & Region & Zone & $\begin{array}{c}\text { TPS } \\
\text { Material }\end{array}$ & $\begin{array}{c}\text { Total Area } \\
\left(\mathbf{m}^{2}\right)\end{array}$ \\
\hline 1 & - & Nosecap & 1001 & $\mathbf{A C C}$ & 0.356 \\
\hline 2 & 33506 & $10 \%$ Body (Wind) & $1020+1050$ & AETB/TUFI & 12.596 \\
\hline 3 & 43219 & $25 \%$ Body (Wind) & $1021+1051$ & CRI & 21.868 \\
\hline 4 & 35179 & 50\% Body (Wind) & 1022 & CRI & 22.558 \\
\hline 5 & 35806 & $75 \%$ Body (Wind) & 1023 & CRI & 62.560 \\
\hline 6 & 24080 & 10\% Body (Lee) & 1010 & CRI & 14.33 \\
\hline 7 & 24834 & 25\% Body (Lee) & 1011 & FRSI & 24.936 \\
\hline 8 & 25775 & 50\% Body (Lee) & $1012,1013,1002$ & FRSI & 146.950 \\
\hline 9 & 8624 & Wing L/E & $30 \%(2101+2201)$ & $\mathbf{A C C}$ & 4.970 \\
\hline $\mathbf{1 0}$ & 9742 & Wing L/E & $30 \%(2101+2201)$ & ACC & 4.970 \\
\hline 11 & 16785 & Wing Acreage (W) & $5 \%(2200)$ & AETB/TUFI & 1.990 \\
\hline 12 & 10645 & Strake & $40 \%(2101+2201)$ & AETB/TUFI & 6.626 \\
\hline 13 & 2054 & Vertical Tail L/E & $8101+8201$ & AETB/TUFI & 5.494 \\
\hline 14 & 37383 & $\begin{array}{l}\text { Wing Acreage } \\
50 \% \text { Chord }(\mathrm{L})\end{array}$ & $78 \%(2100)+8100+8200$ & FRSI & 40.312 \\
\hline 15 & 37429 & $\begin{array}{c}\text { Wing } \\
\text { Acreage } 25 \% \text { Chord (L) }\end{array}$ & $22 \%(2100)$ & AETB/TUFI & 8.406 \\
\hline 16 & 16840 & $\begin{array}{l}\text { Wing Acreage } \\
50 \% \text { Chord }(W)\end{array}$ & 95\%(2200) & CRI & 37.816 \\
\hline
\end{tabular}

Table 2. Summary of body point TPS material and temperature limits

For the thermal optimization metamodels are developed based on the FIAT simulation results. Sixteen metamodels are developed, one for each section of the TPS. The metamodels link the ten design variables from the trajectory analysis with the optimal thickness that results from the FIAT optimization. In this work the metamodels are created using the Kriging method. Soak out conditions are considered in the FIAT simulations. The FIAT code is utilized to compute the optimal thickness for a given set of trajectory design variables while at the same time temperature limits on the bondline surface and locations within the graphite and the core material of the structure are applied. The trajectory variables determine the Mach number, angle of attack, and dynamic pressure time histories. Based on these time histories an aerothermal database is utilized to compute the input environment for FIAT. The CBAERO code is run over an expected range of flight Mach numbers, Angles-of-Attack and free-stream dynamic pressures that will span the trajectory envelope, generating an aerothermal database for the given vehicle configuration. The convective heating environments at each body point are generated by interpolating the aerothermal database as a function of Mach number, angle-of-attack and dynamic pressure as function of time. The resulting convective environments file which is used as input for the FIAT analysis consists of the time history of the recovery enthalpy, the convective film transfer coefficient and the surface pressure. The trajectory optimization and the thermal optimization are linked within the multidiscipline analysis in order to exchange information and facilitate the interaction between the two discipline level optimizations.

\section{Structural Optimization}

For the structural discipline optimization the MSC/Nastran SOL200 is used. The structural finite element model and the locations associated with the twenty seven design variables are presented in Figure 4. This is a coarse finite element model since only stresses from global loading conditions are considered in this work. A description of the twenty seven design variables is presented in Table 3. Four loading conditions are considered in this analysis: the aerodynamic pressure loads corresponding to the $\max (\mathrm{Q}), \max (\mathrm{Q} A l p h a)$, and $2.5 \mathrm{G}$ conditions, and the thrust load. For the hypersonic/supersonic phase of the re-entry trajectory, the surface pressures are generated over the entire surface of the TSTO Upper Stage vehicle by interpolation of the aerodynamic/aerothermodynamic database generated by CBAERO. Maximum free-stream dynamic pressure and the maximum dynamic pressure times angleof-attack occur during the supersonic portion of the entry trajectory. The $2.5 \mathrm{G}$ turn occurs during the final landing approach phase of the flight at a subsonic mach number of 0.5. The surface pressure at this flight condition come from interpolation of an aerodynamic database generated using the NASA Ames Research Center code CART3D [31]. CART3D is an Euler CFD code for computing subsonic, trans-sonic and low supersonic flow over arbitrary aircraft configurations. The thrust load is evaluated by considering that the maximum ascent axial load on the TSTO Upper stage vehicle occurs at the end of the powered flight phase, just before main engine cut-off (MECO). The 
rocket engines are throttled back to a lower power setting to limit the axial acceleration of the vehicle. For this design case, the maximum allowable axial acceleration is limited to $5 \mathrm{~g}$ 's, hence setting the total engine thrust at five times the MECO weight. Although all four loading conditions are considered, the loading originating from the $\operatorname{Max}(\mathrm{Q})$ and $\operatorname{Max}(\mathrm{Q} A l p h a)$ has no influence on the optimization results.
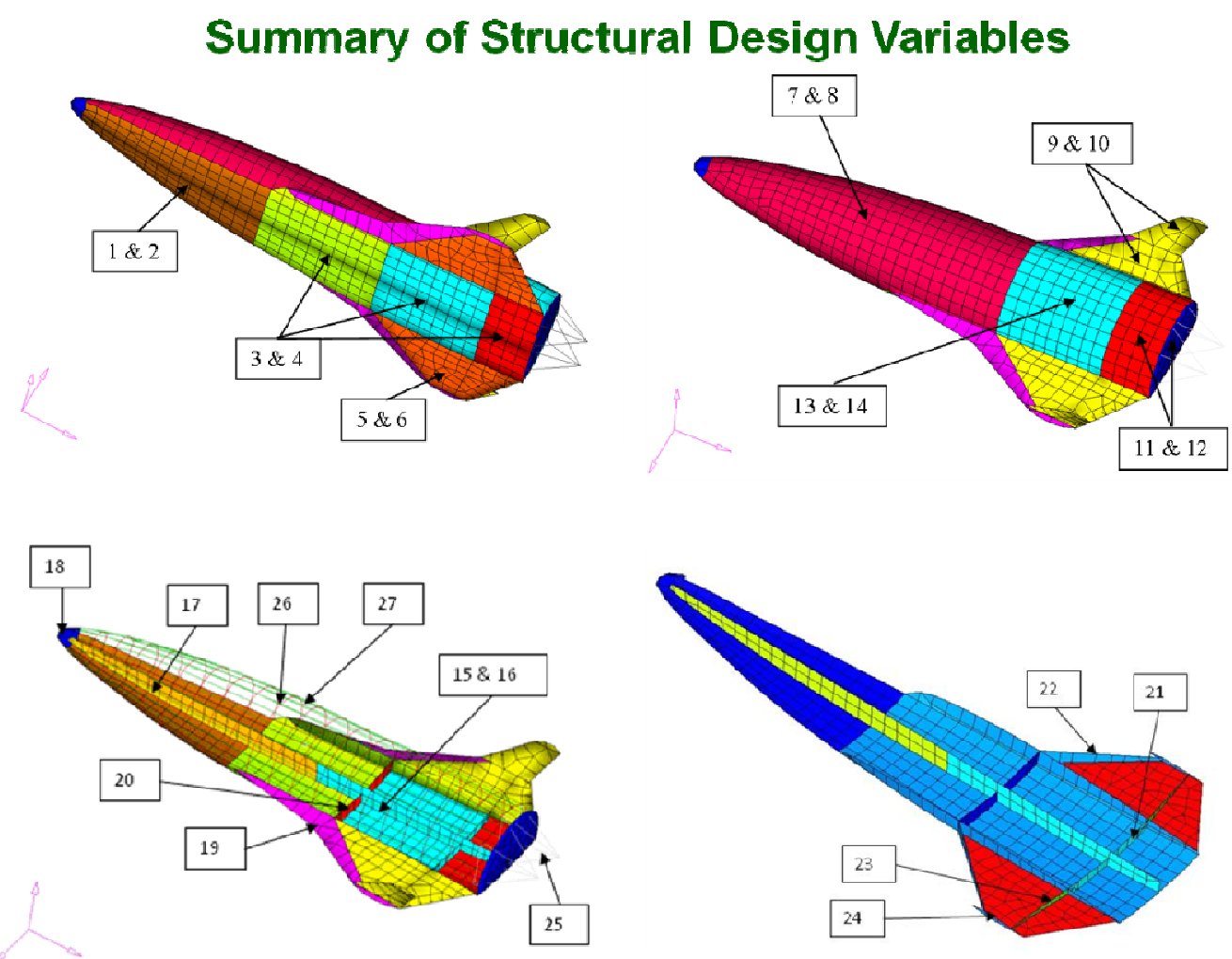

Figure 4. Finite element model used in structural optimization and locations associated with the twenty seven design variables

\begin{tabular}{|c|l|l|}
\hline DV \# & \multicolumn{1}{|c|}{ Material Card } & \multicolumn{1}{|c|}{ Description } \\
\hline $1 \& 2$ & PCOMP 1001 GR \& AL & Front Fuselage Bottom \\
\hline $3 \& 4$ & PCOMP 1002, 1016,1019 GR \& AL & Aft-fuselage Bottom + Wing root closure \\
\hline $5 \& 6$ & PCOMP 1003 GR \& AL & Lower Surface of Wings \\
\hline $7 \& 8$ & PCOMP 1004 GR \& AL & Upper Surface of Fore Fuselage \\
\hline $9 \& 10$ & PCOMP 1005 GR \& AL & Upper Surface of Wing and Rudder \\
\hline $11 \& 12$ & PCOMP 1009, 1020 GR \& AL & Rear End \& Upper Surface of Aft Fuselage \\
\hline $13 \& 14$ & PCOMP 1017 GR \& AL & Upper Surface of Mid-Fuselage \\
\hline $15 \& 16$ & PCOMP 1018 GR \& AL & Aft Center Keel \\
\hline 17 & PSHELL 1006 & Fore Center Keel \\
\hline 18 & PSHELL 1007 & Nose Cap \\
\hline 19 & PSHELL 1008 & Wing Leading Edge \\
\hline 20 & PSHELL 1021 & Front Carry-Through \\
\hline 21 & PSHELL 1022 & Rear Carry-Through \\
\hline 22 & PSHELL 1023 & Front Spar \\
\hline 23 & PSHELL 1024 & Rear Spar \\
\hline 24 & PSEHLL 1025 & Wing-tip Rib \\
\hline 25 & PBARL 1010 & Truss Tube for Thrust Structure \\
\hline 26 & PBEAML 1 & PBEAML BOX -Ring Frame \\
\hline 27 & PBEAML 2 & PBEAML BOX -Longitudinal Frame \\
\hline
\end{tabular}

Table 3. Description of the twenty seven structural design variables 
Constraints on the stresses and strains in the graphite layers and AL core material are imposed during the structural optimization conducted by Nastran SOL200. The Nastran optimization checks for violation of the constraints; additionally, a code provided by NASA Ames processed the Nastran results after the optimization was completed and offered an additional check for the constraints.

A main issue for the structural optimization is that the results depend on the starting point of the optimization. Thus, the level of improvement in the structural weight depends on the starting point of the optimization. In order to address this issue, a customized algorithm is developed and used. The flow chart of this algorithm is presented in Figure 5. Since the initial point for the design variables has an influence on the optimization results, 100 different starting points are created through a random number generator and an optimization analysis is performed for each starting point. Out of all the optimal solutions the one which produces the smallest weight is identified. Then, an iterative process is executed where the optimal solution with the smallest weight comprises the starting point for the optimization. Each time that the optimization is completed, the new optimum configuration is set as the starting point for the next optimization analysis. The process is considered to converge in the optimal solution when there is no further weight reduction between two successive iterations.

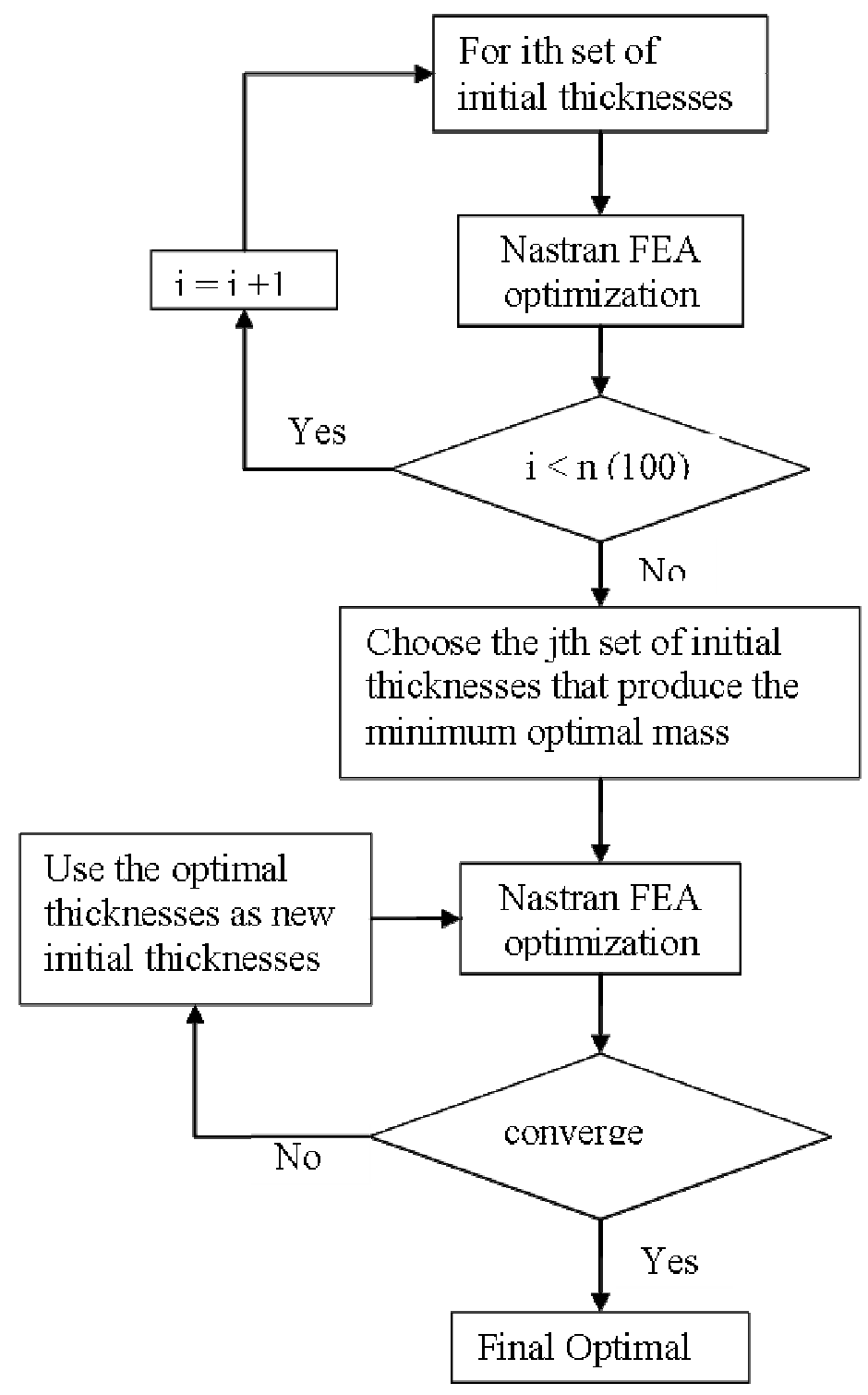

Figure 5. Flow chart of optimization algorithm used for the structural optimization 


\section{Results of Design Optimization Analysis}

A deterministic analysis is performed first without considering any uncertainty during the optimization. Then uncertainties are assigned in three design variables of the trajectory analysis and in parameters associated with the strength of the graphite, the thrust load, and the $2.5 \mathrm{G}$ load and the optimization analysis is repeated. Uncertainties are considered in the $2^{\text {nd }}$ and $3^{\text {rd }}$ angles of attack and in the $3^{\text {rd }}$ bank angle. Thru a sensitivity analysis these three design variables are identified to be the most influential to the optimization results. The uncertainty is introduced in these three angles as a normal distribution with a sigma of 0.25 degrees. The uncertainty in the strength of the graphite is defined as a uniform distribution between $50 \%$ and $100 \%$ of the nominal value. For the thrust load a normal distribution is considered with a variation of + or $-5 \%$ as 3 sigma. For the $2.5 \mathrm{G}$ load a normal distribution is considered with a variation of + or $-0.5 \mathrm{G}$ as 3 sigma. The results from the optimization without and with uncertainty are presented in this section. All the results which are presented here are computed using the actual solvers. For the trajectory and for the structural computations the actual solvers are always utilized during the optimization process. Metamodels are used for the FIAT analysis during the optimization process. In the summary of the results the actual solvers are used for the FIAT simulations once the optimal trajectory parameters have been identified.

Figure 6 presents the results for the bank angle schedules and the angle of attack schedules from the analysis without uncertainty and with uncertainty. Adjustments are made in the trajectory design variables (particularly the bank angle schedule) in the presence of uncertainty in order to satisfy the trajectory constraints and the thermal analysis constraints with a 98\% reliability level in the presence of uncertainty. Table 4 summarizes the results for the trajectory optimization without and with uncertainty. The label "MDO" is used to indicate results from the deterministic analysis and the label "MDOU" indicates results from the optimization in the presence of uncertainty. In the parts of the Table which present the values of the constraints, the MDOU column contains values for the constraints that correspond to the $98 \%$ reliability level. It can be observed that the presence of uncertainty does not allow the optimizer to reduce the objective function of the total heat load as much as the simulation without uncertainty does, in order to introduce the necessary margin of safety in the various constraints. The most prominent constraints in both sets of optimizations appear to be the maximum temperature at body points \#1, \#3, \#4, $\# 5, \# 10, \# 14, \# 16$; and the constraint in the required cross range. As a point of reference indicating the level of improvement achieved by the optmization analysis, the original trajectory demonstrated a heat load of $\sim 79,000$ $\mathrm{J} / \mathrm{cm}^{\wedge} 2$.
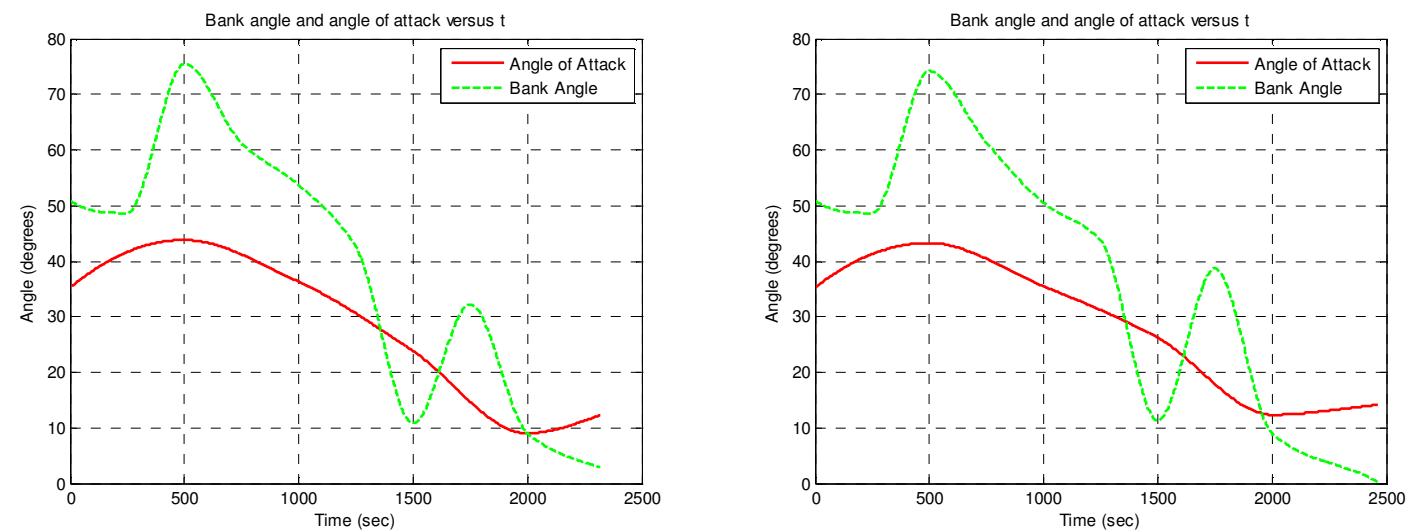

Figure 6. Trajectory summaries for the bank angle schedule and the angle of attack schedule for the trajectories optimized without uncertainty (left) and under uncertainty (right) 


\begin{tabular}{|l|l|l|}
\hline & MIDO & MIDOU \\
\hline Total Heat Load (J/cm 2) & $7.0560 \mathrm{E}+004$ & $7.5850 \mathrm{E}+00+$ \\
\hline
\end{tabular}

\begin{tabular}{|l|c|c|r|}
\hline Body point $\#$ & NIIO & MIIOU & \multicolumn{1}{l|}{$\begin{array}{l}\text { Temp } \\
\text { Limit }\end{array}$} \\
\hline 1 & $2.0000 \mathrm{e}+003$ & $1.9979 \mathrm{e}+003$ & 2000 \\
\hline 2 & $1.4557 \mathrm{e}+003$ & $1.4+117 \mathrm{e}+003$ & 1650 \\
\hline 3 & $1.3831 \mathrm{e}+003$ & $1.3661 \mathrm{e}+003$ & 1400 \\
\hline 4 & $1.3738 \mathrm{e}+003$ & $1.3579 \mathrm{e}+003$ & $1+00$ \\
\hline 5 & $1.3805 \mathrm{e}+003$ & $1.3583 \mathrm{e}+003$ & 1400 \\
\hline 6 & $6.5737 \mathrm{e}+002$ & $6.5056 \mathrm{e}+002$ & 1400 \\
\hline 7 & $6.9170 \mathrm{e}+002$ & $6.89+1 \mathrm{e}+002$ & 750 \\
\hline 8 & $6.1306 \mathrm{e}+002$ & $6.2640 \mathrm{e}+002$ & 750 \\
\hline 9 & $1.7585 \mathrm{e}+003$ & $1.7585 \mathrm{e}+003$ & 2000 \\
\hline 10 & $1.9448 \mathrm{e}+003$ & $1.9235 \mathrm{e}+003$ & 2000 \\
\hline 11 & $1.5011 \mathrm{e}+003$ & $1.4657 \mathrm{e}+003$ & 1650 \\
\hline 12 & $1.2590 \mathrm{e}+003$ & $1.2299 \mathrm{e}+003$ & 1650 \\
\hline 13 & $1.1588 \mathrm{e}+003$ & $1.1943 \mathrm{e}+003$ & 1650 \\
\hline 14 & $7.4102 \mathrm{e}+002$ & $7.3081 \mathrm{e}+002$ & 750 \\
\hline 15 & $7.7325 \mathrm{e}+002$ & $7.5911 \mathrm{e}+002$ & 1650 \\
\hline 16 & $1.3875 \mathrm{e}+003$ & $1.3397 \mathrm{e}+003$ & 1400 \\
\hline
\end{tabular}

\begin{tabular}{|l|c|c|r|}
\hline Description & MDO & MDOU & Limit \\
\hline Max G & $1.1960 \mathrm{e}+000$ & $1.2980 \mathrm{e}+000$ & 3.0 \\
\hline Max Q (psf) & $5.0000 \mathrm{e}+002$ & $3.3375 \mathrm{e}+002$ & 500.0 \\
\hline MaxQA (psf-degrees) & $5.0026 \mathrm{e}+003$ & $5.0038 \mathrm{e}+003$ & 8000.0 \\
\hline Cross Range (11.1m) & $1.2000 \mathrm{e}+003$ & $1.2055 \mathrm{e}+003$ & 1200.0 \\
\hline
\end{tabular}

Table 4. Summary of results for optimal trajectory without and with uncertainty

The results for the thermal analysis discipline are summarized in Table 5. The initial thicknesses for the TPS design, along with the lower and upper bounds, were provided by NASA Ames, and the improvement achieved by the optimization uses the original configuration as a point of reference. The Table contains two columns of results labeled as "MDO" (optimization without uncertainty) and "MDOU" (optimization with uncertainty). As it can be observed the MDOU results provide a more conservative (higher thicknesses) solution for the optimal TPS design. This is due to the more severe heat environments that can get created from the presence of uncertainty in the three design variables of the trajectory simulation. In previous work where the same multidiscipline environment was used to design the TPS of an Apollo type vehicle under uncertainty, the ability of the optimal design to satisfy the constraints in the presence of uncertainty was checked through a Monte Carlo simulation [25]. Therefore, such checks are not conducted for the analysis presented in this paper. 


\begin{tabular}{|c|c|c|c|}
\hline & Initial & $\mathrm{MLO}$ & $\mathrm{MDOU}$ \\
\hline Iotal mass (lbs) & $4.7797 \mathrm{e}+003$ & $4.0624 \mathrm{e}+003$ & $4.2214 \mathrm{e}+003$ \\
\hline
\end{tabular}

\begin{tabular}{|l|c|r|r|r|l|}
\hline & Inital & \multicolumn{1}{c|}{ LB } & \multicolumn{1}{c|}{ UB } & \multicolumn{1}{c|}{ MDO } & MDOU \\
\hline 1 & 3.5 & 0.25 & 8.0 & 5.9629 & 6.1343 \\
\hline 2 & 2.0 & 0.375 & 6.0 & 3.0078 & 3.1328 \\
\hline 3 & 2.0 & 0.25 & 6.0 & 2.5547 & 2.6250 \\
\hline 4 & 2.0 & 0.25 & 6.0 & 1.4463 & 1.4941 \\
\hline 5 & 2.0 & 0.25 & 6.0 & 1.4292 & 1.5215 \\
\hline 6 & 2.0 & 0.25 & 6.0 & 0.4448 & 0.4893 \\
\hline 7 & 0.5 & 0.25 & 2.0 & 0.4297 & 0.4473 \\
\hline 8 & 0.5 & 0.25 & 2.0 & 0.3301 & 0.3323 \\
\hline 9 & 3.5 & 0.25 & 6.0 & 5.4385 & 5.6338 \\
\hline 10 & 3.5 & 0.25 & 6.0 & 5.1162 & 5.2773 \\
\hline 11 & 2.0 & 0.375 & 6.0 & 2.3984 & 2.5156 \\
\hline 12 & 3.5 & 0.25 & 6.0 & 3.2080 & 3.2905 \\
\hline 13 & 2.0 & 0.375 & 6.0 & 1.4351 & 1.6382 \\
\hline 14 & 0.5 & 0.25 & 2.0 & 0.2500 & 0.2500 \\
\hline 15 & 2.0 & 0.375 & 6.0 & 0.4543 & 0.4956 \\
\hline 16 & 2.0 & 0.25 & 6.0 & 1.8701 & 1.9487 \\
\hline
\end{tabular}

Table 5. Summary of the results from the thermal discipline of the multi-discipline optimization

The results from the structural discipline are summarized in Table 6 and in Figures 7-10. The thicknesses are treated as continuous design variables. The initial lower and upper bounds for the twenty seven design variables along with their initial values were provided by NASA Ames. The improvements in reducing the mass of the structure are referenced with respect to the starting point for the optimization. Results for the mass reduction are presented with and without the internal concentrated non-structural masses of the vehicle that are present in the structural analysis in order to capture the weight related loading effects. As it can be observed a significant weight reduction can be achieved in the structure of the vehicle (under the set of the four loads that are considered in this study). Out of all sources of uncertainty, the main one for driving up the weight of the structure is the uncertainty considered in the strength of the graphite material. In the presence of uncertainty thicknesses are increased in order to compensate for the reduced strength of the structure. For certain sandwich panels where bending is the primary form of loading, the thickness of the aluminum core is increased much more than the thickness of the graphite in order to increase the overall moment of inertia of the cross section by moving the graphite section further away from each other. Figures 7 and 8 present the distribution of the thicknesses of the graphite and of the aluminum core, respectively for the deterministic analysis. The results are presented in a non-dimensional manner with respect to the original thickness in each section. Therefore, value equal to one indicate no changes, values higher than one indicate increase in the thickness, and values less than one indicate a reduction in the thickness. Similar results are presented in Figures 9 and 10 for the thickness distribution identified by the multi-discipline optimization with uncertainty. 


\begin{tabular}{|l|l|l|l|}
\hline & Initial & MDO OPT & MDOU \\
\hline Total structure mass (lbs) & 94785 & 74156 & 84610 \\
\hline Without CONM2 (lbs) & 38337 & 17708 & 28162 \\
\hline
\end{tabular}

\begin{tabular}{|c|c|c|c|c|c|}
\hline \multirow[t]{2}{*}{$\overline{D V} \ddot{=}$} & \multicolumn{5}{|c|}{ Thicknesses (inch) } \\
\hline & Lower Bound & Upyer Bound & Luitial & $\mathrm{MDO}$ & MIOU \\
\hline 1 & 0.005 & 0.035 & 0.0052 & 0.005000 & 0.605010 \\
\hline 2 & 1.000 & 4.000 & 2.000 & 1.005070 & 1.607060 \\
\hline 3 & 0.0025 & 0.035 & 0.0052 & 0.006040 & 0.609060 \\
\hline 4 & 1.000 & 4.000 & 2.000 & 1.140600 & 1.865930 \\
\hline 5 & 0.002 & 0.035 & 0.0052 & 0.002750 & 0.608790 \\
\hline 6 & 0.750 & 4.000 & 1.000 & 0.755100 & 3.678950 \\
\hline 7 & 0.0025 & 0.035 & 0.0052 & 0.002500 & 0.606370 \\
\hline 8 & 1.000 & 4.000 & 1.500 & 1.000000 & 1.600000 \\
\hline 9 & 0.002 & 0.035 & 0.0052 & 0.005200 & 0.607840 \\
\hline 10 & 0.750 & 4.000 & 0.750 & 0.751300 & 0.768880 \\
\hline 11 & 0.005 & 0.035 & 0.0052 & 0.005020 & 0.009930 \\
\hline 12 & 1.000 & 4.000 & 1.500 & 1.755790 & 1.689860 \\
\hline 13 & 0.002 & 0.035 & 0.0052 & 0.002000 & 0.603760 \\
\hline 14 & 1.000 & 4.000 & 1.500 & 1.010890 & 3978660 \\
\hline 15 & 0.002 & 0.035 & 0.0052 & 0.002000 & 0.603900 \\
\hline 16 & 1.000 & 4.000 & 2.000 & 1.006170 & 1.392550 \\
\hline 17 & 0.250 & 2.500 & 2.000 & 0.250000 & 0.601740 \\
\hline 18 & 0.200 & 1.500 & 0.300 & 0.253100 & 0.250260 \\
\hline 19 & 0.260 & 1.500 & 0.300 & 0.380080 & 0.799830 \\
\hline 20 & 0.250 & 3.500 & 2.000 & 0.591650 & 0.632030 \\
\hline 21 & 0.250 & 3.500 & 2.000 & 0.958350 & 1.806870 \\
\hline 22 & 0.250 & 3.500 & 2.000 & 0.734740 & 1.6377600 \\
\hline 23 & 0.250 & 3.500 & 2.000 & 1.622280 & 3.498450 \\
\hline 24 & 0.250 & 3.500 & 2.000 & 0.809030 & 1.105220 \\
\hline 25 & 2.260 & 4.000 & 2.500 & 2.371460 & 2.400160 \\
\hline 26 & 0.100 & 0.800 & 0.500 & 0.100000 & 0.100000 \\
\hline 27 & 0.100 & 0.800 & 0.375 & 0.100000 & 0.108250 \\
\hline
\end{tabular}

Table 6. Summary of optimization results for structural discipline

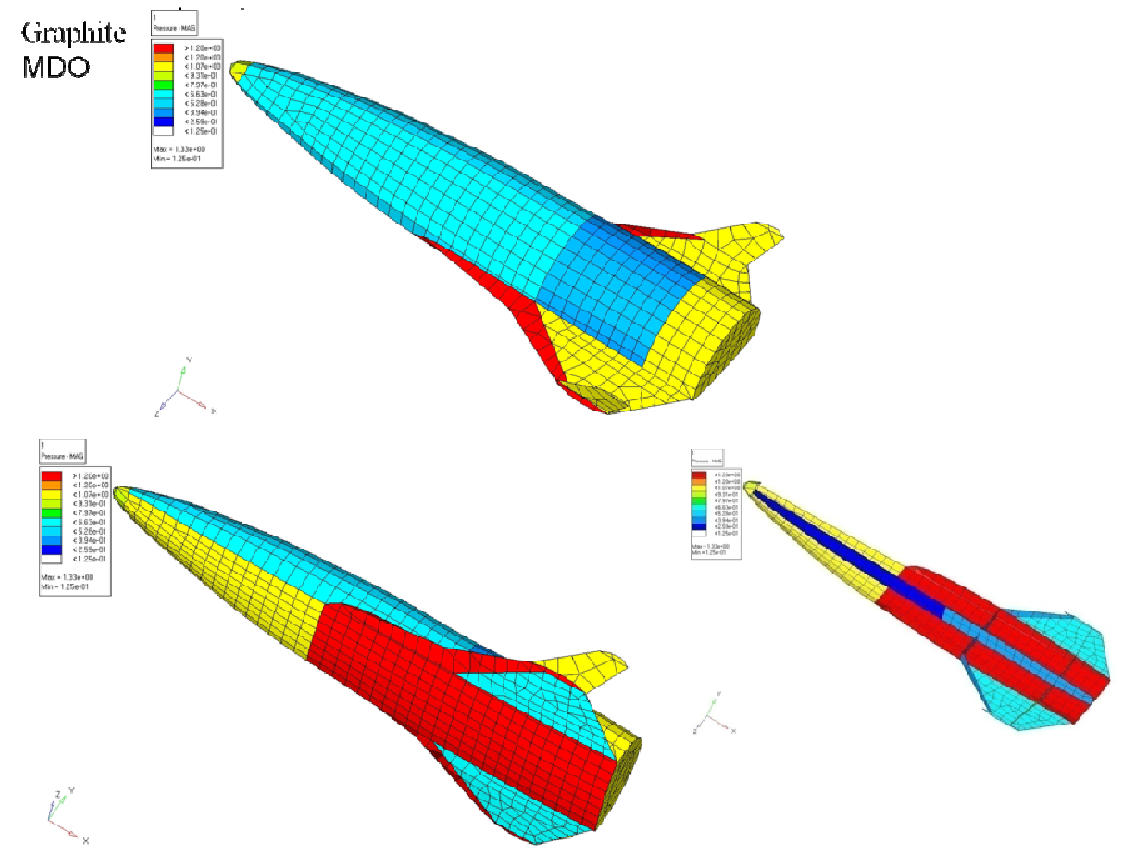

Figure 7. Distribution of optimized graphite thickness from the deterministic analysis

11

American Institute of Aeronautics and Astronautics 


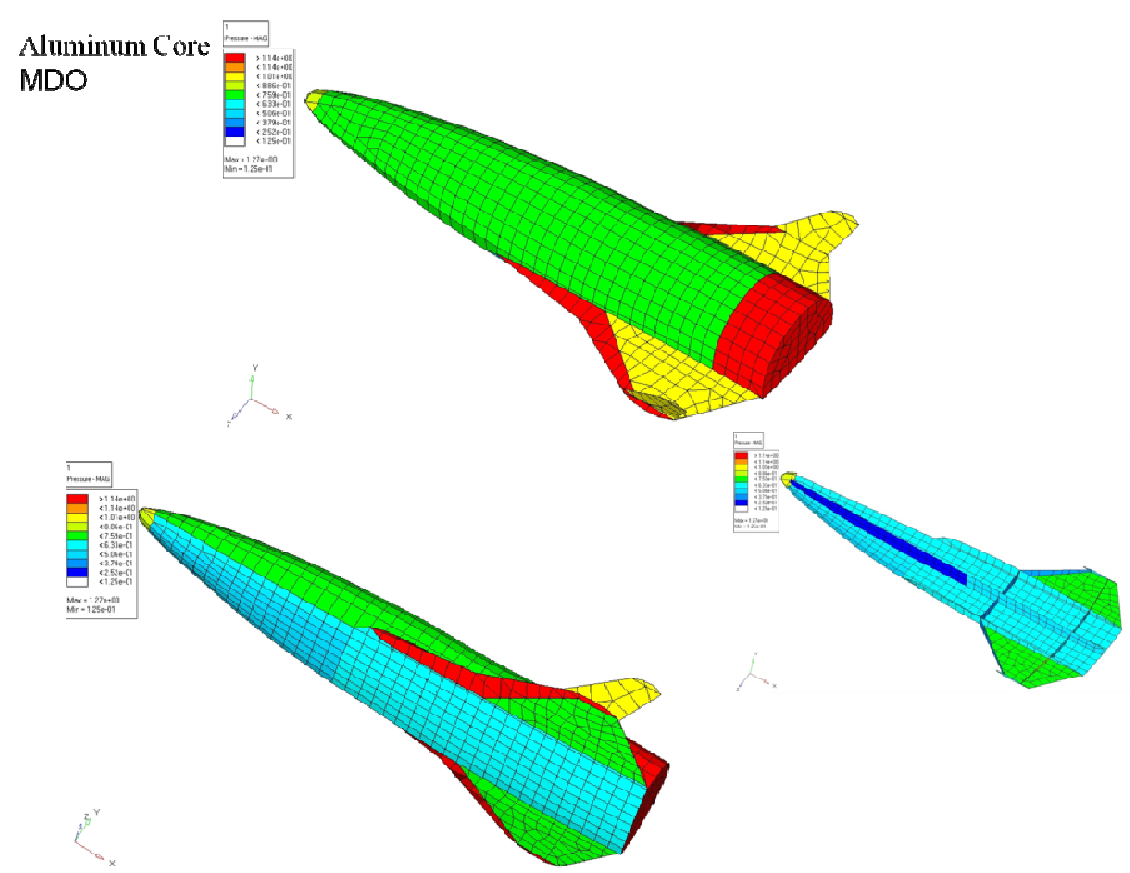

Figure 8. Distribution of optimized aluminum core thickness from the deterministic analysis

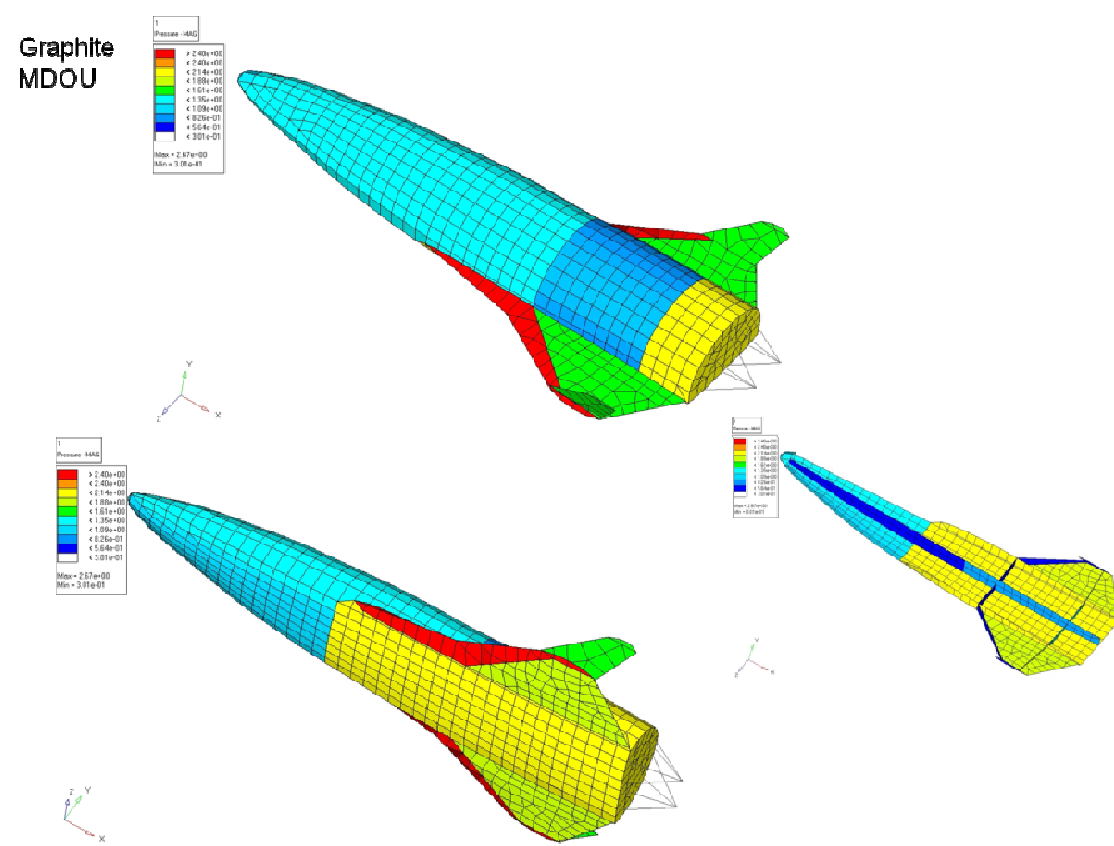

Figure 9. Distribution of optimized graphite thickness from the analysis with uncertainty 


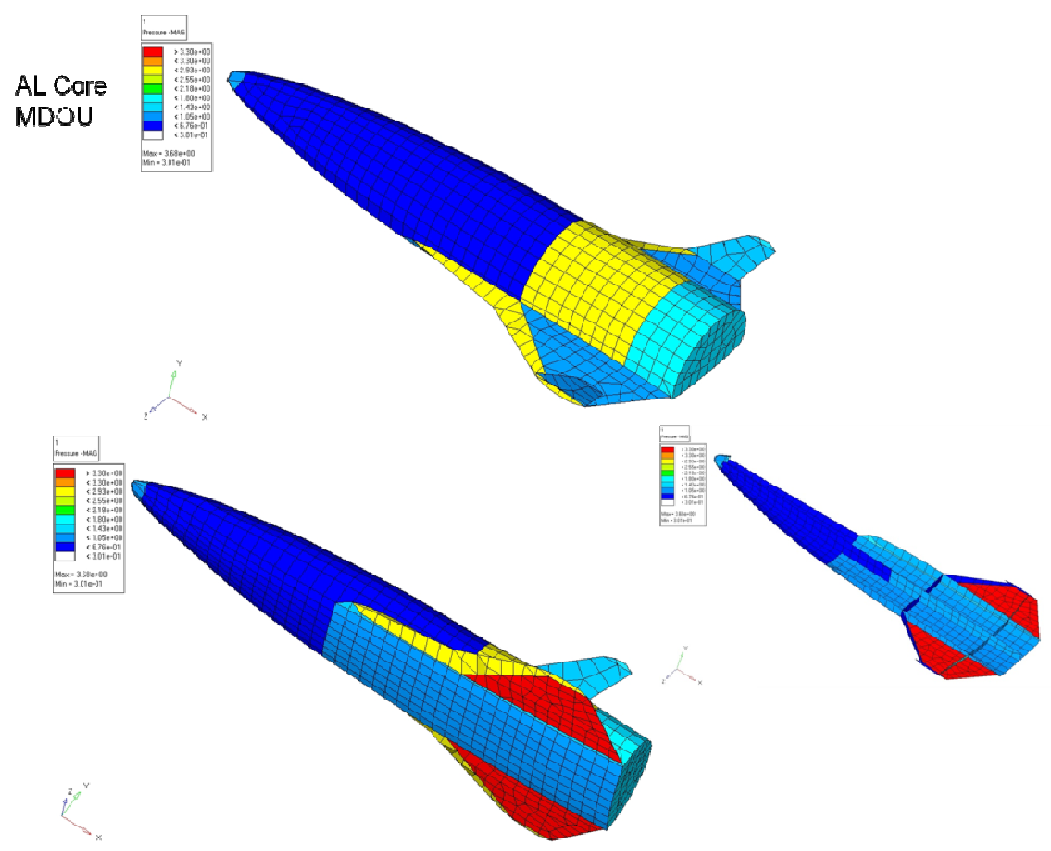

Figure 10. Distribution of optimized aluminum core thickness from the analysis with uncertainty

\section{Conclusions}

The work presented in this paper demonstrates how a flexible optimization environment [32] that links seamlessly multiple solvers; drives in parallel multiple optimization analyses while automating the exchange of data between them; and allows to account for uncertainty in the decision making process can be utilized for the multidiscipline design of a hypersonic vehicle. Additionally, the capability of creating metamodels and employing them in the multi-discipline design computations is available and employed in this work. Understanding the behavior of the optimization in each discipline is important in terms of the interdependency of different disciplines to the same set of design variables. Conducting a sensitivity analysis helps identify the important design variables and the important parameters for assigning uncertainty. There is no single recipe for formulating the optimization statement; instead the structure of the optimization must reflect the physics of the particular problem of interest. It is advisable to utilize metamodels when the computational savings will be significant, instead of trying to create them for all objective functions and all constraints. Capturing uncertainty is important and allows for all disciplines to adjust their performances in order to meet the probabilistic constraints, thus, leading to superior performance under uncertainty.

\section{Acknowledgments}

The technical assistance provided by the following individuals from NASA Ames Research Center was valuable for the completion of the work presented in this paper: Jeff Bowles, Loc Huynh, Alberto Makino, Unmeel Mehta, Hiro Miura, and David Saunders. This work was part of the Phase II SBIR contract NNA06AA05C.

\section{References}

1 Vatistas D.O. Stanley, W.C. England, R.A. Lepsch, M. Mcmillin, K.E. Wurster and R.W. Powell, "Rocket-Powered SingleStage Vehicle Configuration Selection and Design," AIAA Paper 93-1053, Feb. 1993.

2 D.O. Stanley, W.C. Engeland, and R.A. Lepsch, "Propulsion System Requirements for Reusable Single-Stage-to-Orbit Rocket Vehicles," AIAA Paper 92-3504, July 1992.

3 P.V. Tartabini, R.A. Lepsch, J.J. Korte K.E. Wurster, “A Multidisciplinary Performance Analysis of a Lifting-Body SingleStage-to-Orbit Vehicle," AIAA Paper 2000-1045, 38 ${ }^{\text {th }}$ Aerospace Sciences Meeting \& Exhibit, January 2000, Reno, NV.

4 J. Riehl, C. Trefny, D. Kosareo, “SRM-Assisted Trajectory for the GTX Reference Vehicle,” NASA / TM -2000-211599.

5 J.T. Betts, "Using Sparse Nonlinear Programming to Compute Low Thrust Orbit Transfers," The Journal of the Astronautical Sciences, Vol. 41, No. 3, July-September 1993, pp 349-371.

$6 \quad$ J.T. Betts, "Optimal Interplanetary Orbit Transfers by Direct Transcription," The Journal of the Astronautical Sciences, Vol. 42, No. 3, July-September 1994, pp 247-268. 
7 P.C. Calhoun and E.M. Queen, "Entry Vehicle Control System Design for the Mars Smart Lander," AIAA-2002-4504, AIAA Atmospheric Flight Mechanics Conference and Exhibit, 5-8 August 2002, Monterey, California.

8 C.E. Cockrell, Jr., "Aerosciences, Aero-Propulsion and Flight Mechanics Technology Development for NASA's Next Generation Launch Technology Program," AIAA 2003-6948, $12^{\text {th }}$ AIAA International Space Planes and Hypersonic Systems and Technologies Conference, December 15-19, 2003, Norfolk, Virginia.

9 C.G. Miller, "Aerothermodynamic Flight Simulation Capabilities for Aerospace Vehicles," AIAA-98-2600, 20 ${ }^{\text {th }}$ AIAA Advance Measurement \& Ground Testing Technology Conference, June 1998, Albuquerque, New Mexico.

10 B.R. Hollis, "Experimental and Computational Aerothermodynamics of a Mars Entry Vehicle," NASA Contractor Report 201633, December 1996.

11 C.G. Miller, "Langley Hypersonic Aerodynamic/Aerothermodynamic Testing Capabilities - Present and Future," AIAA Paper 90-1376, 1990.

12 J.A. Dec, J.F. Gasbarre, B.E. George, “Thermal Analysis and Correlation of the Mars Odyssey Spacecraft's Solar Array During Aerobraking Operations," AIAA 2002-4536, AIAA/AAS Astrodynamics Conference, August 2002, Monterey, California.

13 R.M. Amundsen, J.A. Dec, M.C. Lindell, “Thermal Analysis Methods for an Earth Entry Vehicle," Eleventh Thermal and Fluids Analysis Workshop, August 21-25, 2000, Cleveland, Ohio.

14 R.M. Amundsen, J.A. Dec, R.A. Mitcheltree, M.C. Lindell, R.A. Dillman, "Preliminary Thermal Analysis of a Mars Sample Return Earth Entry Vehicle," AIAA 2000-2584, 34 ${ }^{\text {th }}$ AIAA Thermophysics Conference, June 19-22, 2000, Denver, Colorado.

15 TPSX, Thermal Protection System and Material Properties Database, February 1998, NASA Ames Research Center (http://asm.arc.nasa.gov/tpsx).

16 S.J. Scotti, C. Clay, M. Rezin, "Structures and Materials Technologies for Extreme Environments Applied to Reusable Launch Vehicles," AIAA 2003-2697, AIAA/ICAS International Air \& Space Symposium and Exposition, July 14-17, 2003, Dayton, Ohio.

17 M.L. Blosser, "Investigation of Fundamental Modeling and Thermal Performance Issues for a Metallic Thermal Protection System Design," AIAA 2002-0503, 40 ${ }^{\text {th }}$ Aerospace Sciences Meeting \& Exhibit, January 2000, Reno, Nevada.

18 M.L. Blosser, "Development of Metallic Thermal Protection Systems for the Reusable Launch Vehicle," NASA TM II 0296, October 1996.

19 D.E. Myers, C.J. Martin, M.L. Blosser, "Parametric Weight Comparison of Advanced Metallic, Ceramic Tile, and Ceramic Blanket Thermal Protection Systems," NASA/TM 2000-210289.

20 R.D. Braun, A.A. Moore, I.M. Kroo, "Use of the Collaborative Optimization Architecture for Launch Vehicle Design," AIAA 1996-1024.

21 S.L. Padula, J.J. Korte, H.J. Dunn, A.O. Salas, "Multidisciplinary Optimization Branch Experience Using iSIGHT Software," NASA/TM 1999-209714.

22 P.V. Tartabini, R.A. Lepsch, J.J. Korte K.E. Wurster, “A Multidisciplinary Performance Analysis of a Lifting-Body SingleStage-to-Orbit Vehicle,” AIAA Paper 2000-1045, 38 ${ }^{\text {th }}$ Aerospace Sciences Meeting \& Exhibit, January 2000, Reno, NV.

23 R.D. Braun, and I.M. Kroo, "Development and Application of the Collaborative Optimization Architecture in a Multidisciplinary Design Environment," AIAA 1995-0704.

24 J. Sun, G. Zhang, N. Vlahopoulos, S-B. Hong, "Multi-disciplinary design optimization under uncertainty for thermal protection system applications," 11th AIAA/ISSMO Multidisciplinary Analysis and Optimization Conference, September 2006, Portsmouth, Virginia, AIAA Paper 2006-7002.

25 N. Vlahopoulos, J. He, "Application of a multidiscipline design optimization approach for designing the thermal protection system of an Apollo type vehicle under uncertainty," 2008 JANNAF Meeting, Orlando FL, December 2008.

26 G. A. Allen, M. J. Wright, P. Gage, “The Trajectory Program (Traj): Reference Manual and User's Guide,” Version 7.2, 20 February 2004, NASA Ames.

27 D. J. Kinney, "Aero-Thermodynamics for Conceptual Design," 42 ${ }^{\text {nd }}$ AIAA Aerospace Sciences Meeting and Exhibit," 5-6 January 2004, Reno, NV.

28 D.J. Kinney, J.A. Garcia, L. Huynh, "Predicted Convective and Radiative Aerothermodynamic Environments for Various Reentry Vehicles using CBAERO," $44^{\text {th }}$ AIAA Aerospace Meeting and Exhibit, 9-12 January 2006, Reno, NV.

29 M.K. McGuire, P. Gage, E.T. Galloway, L. Huyhn, J. Nguyen, J. V. Bowles, R. Windhorst, "Trajectory and Thermal Protection System Design for Reusable Launch Vehicles," $10^{\text {th }}$ AIAA/ISSMO Multidisciplinary Analysis and Optimization Conference, 30 August - 1 September 2004, Albany New York.

30 Y. K. Chen, and F. S. Milos, "Ablation and Thermal Response Program for Spacecraft Heatshield Analysis," Journal of Spacecraft and Rockets, vol. 36, No. 3, 1999, pp. 475-482.

31 Melton, J.E., Berger, M.J., Aftosmis, M.J., “3-D Applications of a Cartesian Grid Euler Method”, AIAA Paper 95-0853, Jan. 1995.

32 NEtwork of oPTimizations UNder uncErtainty (NEPTUNE), Version 2.3, Michigan Engineering Services, LLC. 